\title{
Aerobic Exercise
}

National Cancer Institute

\section{Source}

National Cancer Institute. Aerobic Exercise. NCI Thesaurus. Code C39774.

Sustained exercise that places an increased oxygen demand on the cardiovascular system and promotes an increased efficiency of the body's use of oxygen. 